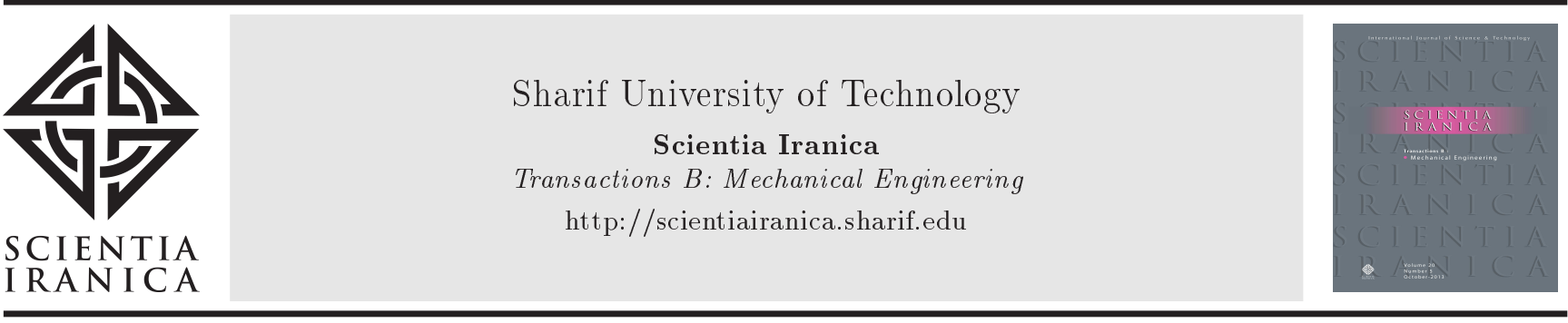

\title{
Numerical investigation into the effect of viscosity on bubble dynamics in a narrow channel
}

\author{
A. Soroureddin ${ }^{a}$, M.T. Shervani-Tabar ${ }^{a, *}$, and H. Aminfar ${ }^{b}$ \\ a. Center for CFD Studies on Heat Engines, Cavitational Flows and Petroleum Industries, Department of Mechanical Engineering, \\ University of Tabriz, Tabriz, P.O. Box 51666-14766, Iran. \\ b. Department of Mechanical Engineering, University of Tabriz, Tabriz, P.O. Box 51666-14766, Iran.
}

Received 16 November 2018; received in revised form 4 March 2019; accepted 2 July 2019

\section{KEYWORDS}

Bubble dynamics;

Boundary element method;

Viscous pressure

correction;

Narrow channel;

Viscous potential flow.

\begin{abstract}
In this paper, dynamic behavior of a vapor bubble inside a narrow channel filled with a viscous liquid is numerically studied. The boundary integral equation method and the procedure of Viscous Correction of Viscous Potential Flow (VCVPF) were employed to obtain the vapor bubble profiles during pulsations inside a narrow channel filled with a viscous liquid. A new method was adopted to consider the effects of viscosity in a viscous liquid flow within the framework of the Green's integral formula together with the modified form of unsteady Bernoulli equation. The reported experimental and numerical results for the problem under investigation were used in the verification of the results of the present work. Numerical results showed that by increasing the viscosity of liquid around the vapor bubble, bubble lifetime increased. They also indicated that for Reynolds numbers with the order of $O\left(10^{3}\right)$, the viscosity effects were extremely reduced. Furthermore, dynamic behavior of bubble in water and oil was investigated at different Reynolds numbers and at different so-called dimensionless channel radii.
\end{abstract}

(C) 2020 Sharif University of Technology. All rights reserved.

\section{Introduction}

Understanding bubble dynamics near the boundary with axial symmetry can be important in industry, medical science, and different natural phenomena. For example, in the oil industry, to improve oil production in reservoirs that are damaged, cavitation bubbles are created near the walls by ultrasound waves [1-3]. In the petrochemical industry, at different stages such as oil processing and oil transport, the creation of Taylor bubbles by two-phase flow in pipes causes slug flow $[4,5]$. Air gun fire produces low-frequency acoustic

\footnotetext{
*. Corresponding author. Tel.: +984133393049; Fax: +984133354153

E-mail addresses: stmech.tab@gmail.com, and msherv@tabrizu.ac.ir (M.T. Shervani-Tabar)
}

doi: $10.24200 /$ sci. 2019.52198 .2589 waves leading to under-sea-level structures, which are important in geology and exploration of oil and gas. In this application, the outflow of high-pressure gas from the main chamber towards the fluid creates a bubble with axial symmetry between the shuttle and the main cylindrical chamber [6,7]. In marine engineering, injection and expansion of compressed gas bubbles in water jet cause net thrust augmentation [810]. In medical science, using embolism technique in therapeutic applications, gas bubbles are created inside the vein by high-frequency sound waves $[11,12]$.

To our knowledge, dynamic behavior of bubble by the Boundary Element Method (BEM) near the boundary with axial symmetry, e.g., in a narrow channel, compared with the rigid boundary or free boundary has rarely been studied in the literature [13-18]. Moreover, in most of the studies related to bubble dynamics inside a narrow channel based on BEM, viscosity effects are ignored. In these researches, the dynamics and bubble 
shape over a period of time, as well as the life of bubble, have been studied along with the geometric parameters of tube. To validate the results, they have chosen fluids with low viscosity like water [19-22]. As a result, their results are not generalizable to viscous fluids without more investigations.

The concept of applying viscosity as an additional pressure term in boundary layer analysis was first proposed by Moore [23]. In order to estimate drag force in a rising bubble, he attempted to incorporate the contribution of viscosity in boundary layer as an additional pressure. Nevertheless, he could not obtain a uniform relationship for the additional pressure. He concluded that to simulate the non-rectilinear motion observed for bubbles larger than about $2 \mathrm{~mm}$, a general $3 \mathrm{D}$ rather than an axi-symmetric model was required. Kang and Leal [24] accurately estimated the drag coefficient for a spherical bubble by calculating the normal stress and integrating it into the bubble surface. In their work, VPC (Viscous Pressure Correction) was estimated by a uniform relationship for the flow with high Reynolds numbers for the first time. Their study showed that without considering the boundary layer and using the irrotational flow solution, VPC could be estimated accurately. In other words, they concluded that VPC was almost independent of vorticity. They also indicated that the drag coefficient up to $O\left(R^{-1}\right)$ depended only on the $O(1)$ vorticity distribution in the fluid.

Joseph [25] obtained the drag value by direct estimation based on Viscous Correction of Viscous Potential Flow (VCVPF) in several hydrodynamic examples. According to their theory, in problems incorporating vorticity layers, VPC can be added to the estimated irrotational pressure. They suggested that the vorticity effects exerted by Unbalanced Irrotational Shear Stress (UISS) could be applied to problem-solving. In their study, for problems with axial symmetry, VPC was considered as s series of surface harmonics. However, they could not propose a uniform relationship for all constant coefficients of the harmonic terms. In particular cases, the constant coefficient of one harmonic term was determined by viscosity loss definition for Viscous Potential Flow (VPF) and VCVPF while other terms were ignored.

Klaseboer et al. [26] investigated the development and deformation of a rising bubble in water at high Reynolds numbers. To simulate the non-rectilinear motion observed for bubbles larger than about $2 \mathrm{~mm}$, a general 3D rather than an axi-symmetric model was required. In this study, the probability of the occurrence of vortex at the downstream flow was not investigated. For numerical simulation, they used BEM and inserted the VPC obtained by Joseph and Wang [27] in the unsteady Bernoulli equation. Joseph and Wang [27] investigated the VPF over a free surface. In their work, the dissipation method was used to study the viscosity effects. An explicit relation was found between pressure correction and irrotational shear stress at the interface. They reported that the drag value obtained by utilizing the dissipation method was equal to $12 \pi \mu \mathrm{aU}$, which was the same as that calculated by using the pressure correction method.

Recently, several researchers have studied VCVPF method to investigate the bubble dynamic behavior near the boundary [28-30]. In all of these studies, VPC has been assumed as a function of normal stress with a constant coefficient at each time step. The coefficient was estimated such that the mechanical energy equation for Navier-Stokes equation was satisfied. However, no appropriate estimation was found in certain moments of the bubble life, because it was assumed that in some points on the bubble surface where the normal stress had large numerical values, the VPC value would be significant, which was not the case. According to the definition, VPC is directly related to UISS value [27].

In this study, the bubble dynamics inside a thin rigid channel are numerically studied by developing a computer code based on the BEM and implementing the VCVPF method for fluids with different viscosities. Also, a new technique is proposed to estimate the VPC parameter at the gas-fluid interface. Accordingly, the value of this parameter at each surface element is predicted so that the power of the traction integral is the same for VPF and VCVPF. In this method, in order to determine $\mathrm{VPC}$, it is not necessary to consider an additional assumption of consistency with the normal stress. In the present work, unlike the previous researches, the assumption of linear relation between VPC and viscous normal stress is not made. Also, the effects of UISS, VPC, and viscous normal stress parameters on the bubble lifetime are investigated. In addition, the expression of equations in discretized form for viscosity parameters on the bubble surface is another distinguishing feature of the present work from other researches. The numerical results obtained for the growth and collapse of bubble inside water in the rigid tube are validated with the experimental data published by $\mathrm{Ni}$ et al. [14]. To the best of our knowledge, experimental results are missing in the literature on bubble dynamics in viscous fluids inside narrow tubes. In this regard, the present numerical results for viscous fluid are compared with the theoretical results reported by Minsier et al. [31] by solving two-dimensional Navier-Stokes equations near the rigid boundary.

\section{Model description}

Figure 1 shows schematics of a bubble with axial symmetry at the center of a thin rigid channel. The 


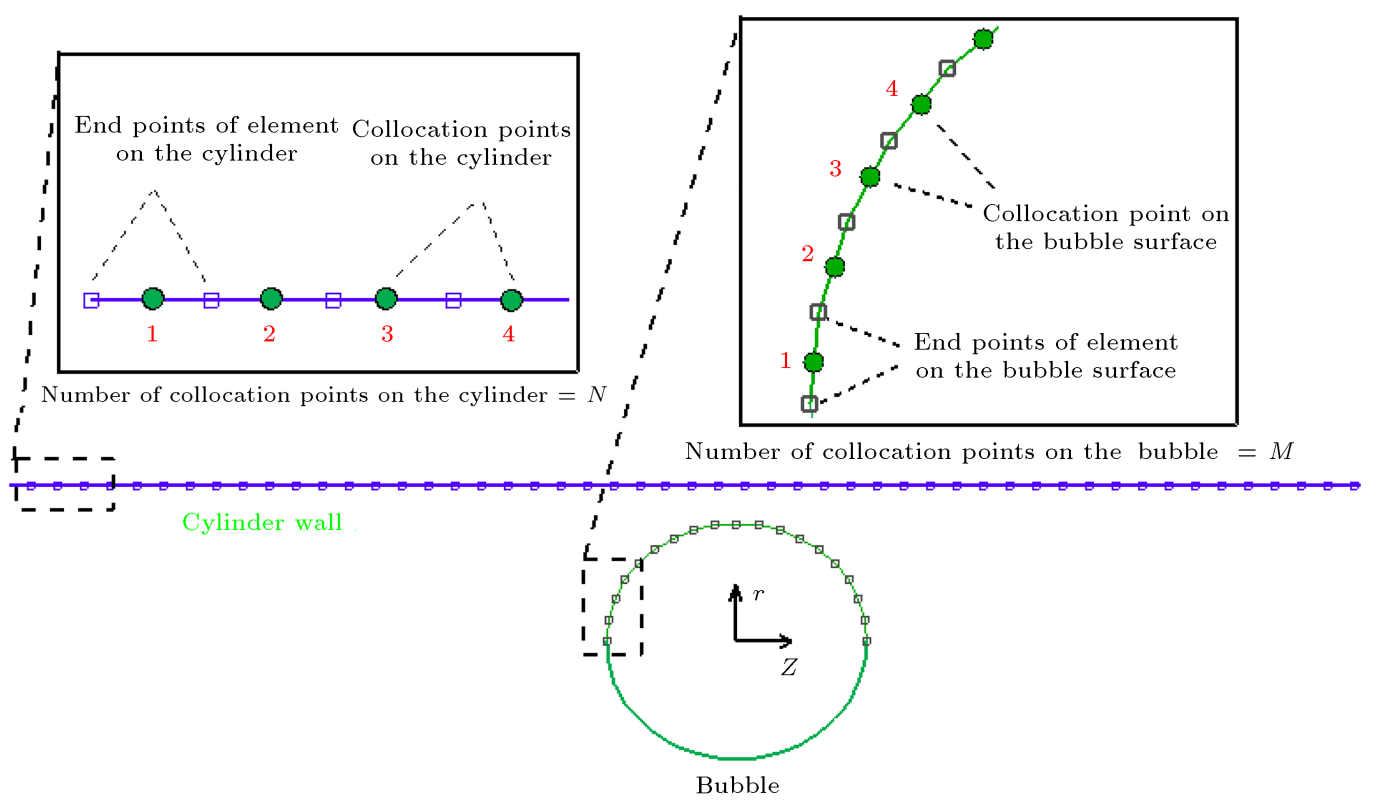

Figure 1. Schematics of the discretized boundaries.

bubble boundary and the wall of the channel are divided to elements considering axial symmetry. In order to prevent imcorporating the effects of other types of boundaries such as free surface in bubble behavior, channel length is assumed very larger than its diameter. The fluid around the bubble is considered Newtonian incompressible. In addition, due to the small size and short life of the bubble, buoyancy force is ignored $[32,33]$. Furthermore, the primary shape of the bubble is considered spherical the radius of which is determined by the Rayleigh-Plesset equation [34].

Integral representation of the solution for the Laplace equation at all points inside and at the boundary of the flow field is obtained from Green's second identity[35].

$$
c(\mathbf{p}) \phi(\mathbf{p})=\int_{\Gamma}\left(\frac{\partial \phi(q)}{\partial n_{q}} G(\mathbf{p}, q)-\phi(q) \frac{\partial G(\mathbf{p}, q)}{\partial n_{q}}\right) d s,
$$

where $\mathbf{p}$ and $q$ are the flow field point and the source point, respectively. In the above equation, $c(\mathbf{p})$ is solid angle, $\phi$ is velocity potential, $G$ is the Green function for the Laplace equation, and $\Gamma$ is the boundary of fluid domain. The Green's integral formula, which governs the potential fluid flow problems in its classical form, is employed as the principal governing equation for the numerical simulation to the hydrodynamic behavior of the vapor bubble inside the viscous liquid filled narrow channel.

\subsection{Boundary conditions}

The fluid inside the bubble is composed of noncondensable gas and saturated vapor at the reference temperature. The gas behavior inside the bubble is ideal and adiabatic [36]. The normal stress balance at the bubble surface is expressed by the Young-Laplace equation as follows [26]:

$$
p_{c}+p_{i g}\left(\frac{R_{i}}{R}\right)^{3 \gamma}-\sigma k+2 \mu \frac{\partial^{2} \phi}{\partial n^{2}}=p_{l}
$$

where $p_{c}$ and $p_{i g}$ are the saturated pressure and the initial gas pressure inside the bubble, respectively. In the above equation, $R_{i}$ is the initial bubble radius, $\sigma$ is the interface surface tension, $k$ is local mean bubble surface curvature, $\mu$ is dynamic viscosity, $p_{l}$ is pressure at the bubble surface, and $\gamma$ is the ratio of specific heat.

For the gas-bubble interface, to create a uniform boundary and to increase estimation accuracy, $M$ number of cubic spline elements with constant physical functions are applied. For the channel wall, $N$ number of linear elements with constant physical functions are considered. The cubic splines are used to discretize the bubble surface. The spline on the first and last nodes of the bubble is clamped. In this way, due to bubble symmetry, the spline on these nodes is tangent to the direction normal to the axis of symmetry:

$$
\frac{\partial r}{\partial l_{j}}=+1, \frac{\partial z}{\partial l_{j}}=0, \quad @ j=1, M
$$

Discretization of the internal wall of the rigid cylinder is continued up to the physical infinity where the pulsation of vapor bubble has a negligible effect on the fluid flow. At the center of each element, at each time scale, the velocity potential at collocation points is estimated by the Lagrangian form of the modified Bernoulli's equation. To represent the distribution of velocity potential on the bubble, cubic spline functions are used. 


\subsection{Viscous Correction of Viscous Potential Flow (VCVPF) method}

The Navier-Stokes equation for an uncompressible viscose fluid with constant properties can be written as [37]:

$$
\rho \frac{\partial \mathbf{U}}{\partial t}+(\mathbf{U} . \nabla) \mathbf{U}=\rho g-\nabla p+\mu \nabla^{2} \mathbf{U}
$$

where $\rho$ is fluid density, $\mathbf{U}$ is velocity vector, $\mathbf{g}$ is body force, and $p$ is pressure.

The equation of mass conservation for an incompressible fluid is expressed as follows [37]:

$$
\nabla \cdot \mathbf{U}=0 .
$$

The velocity vector in the flow field can be divided to irrotational velocity vector and rotational velocity vector by employing Helmholtz decomposition [38].

$$
\mathbf{U}=\mathbf{u}+\mathbf{v}=\nabla \phi+\mathbf{v} \text {. }
$$

By inserting Eq. (6) in Eq. (4), unsteady Bernoulli equation will be achieved:

$$
\begin{aligned}
& \nabla\left(\rho \frac{\partial \phi}{\partial t}+\frac{\rho}{2}|\nabla \phi|^{2}+p+\rho g z\right)+\rho \frac{\partial \mathbf{v}}{\partial t} \\
& \quad+\rho \nabla \cdot[\mathbf{v} \otimes \nabla \phi+\nabla \phi \otimes \mathbf{v}+\mathbf{v} \otimes \mathbf{v}]-\mu \nabla^{2} \mathbf{v}=0
\end{aligned}
$$

in which:

$$
\nabla p_{v c}=\rho \frac{\partial \mathbf{v}}{\partial t}+\rho \nabla \cdot[\mathbf{v} \otimes \nabla \phi+\nabla \phi \otimes \mathbf{v}+\mathbf{v} \otimes \mathbf{v}]-\mu \nabla^{2} \mathbf{v}
$$

In certain hydrodynamic problems such as Poiseuille flow or Hadamard-Rybczynski solution for the flow around a liquid sphere, it is possible to accurately calculate VPC based on Helmholtz decomposition [34]. However, for most of the hydrodynamic problems such as the growth and collapse of bubble inside the channel, no accurate VPC estimation according to Eq. (8) is presented. Therefore, in VCVPF technique, in order to generalize the potential flow method to viscous flows, a value for VPC should be estimated.

Replacing Eq. (8) in Eq. (7) and integrating them, the modified Bernoulli equation will be obtained.

$$
\rho \frac{\partial \phi}{\partial t}+\frac{\rho}{2}|\nabla \phi|^{2}+p+p_{v c}+\rho g z=p_{\infty} .
$$

Dynamic condition on the bubble surface is estimated from the following equation, which is obtained from rearranging Eqs. (3) and (9) based on Lagrangian form.

$$
\begin{aligned}
\rho \frac{D \phi}{D t} & =\frac{1}{2} \rho|\nabla \phi|^{2}+\rho g z+\sigma k+p_{\infty}-p_{c}-p_{i g}\left(\frac{R_{i}}{R}\right)^{3 \gamma} \\
& -2 \mu \frac{\partial^{2} \phi}{\partial n^{2}}-p_{v c}
\end{aligned}
$$

where the terms $2 \mu \partial^{2} \phi / \partial n^{2}$ and $p_{v c}$ indicate the viscous normal stress and $p_{v c}$, respectively. These are terms through which viscosity enters the potential estimations.
Stress at the bubble surface employing Helmholtz decomposition is defined as:

$$
\begin{aligned}
\tau= & -p \mathbf{l}+\mu\left(\nabla \mathbf{U}+\nabla \mathbf{U}^{T}\right)=-p \mathbf{l}+\mu\left(\nabla \mathbf{v}+\nabla \mathbf{v}^{T}\right) \\
& +2 \mu \nabla \otimes \nabla \phi .
\end{aligned}
$$

Viscous rotational shear stress $\mu\left(\nabla \mathbf{v}+\nabla \mathbf{v}^{T}\right)$ at the bubble surface due to low viscosity of gas inside the bubble is almost zero. On the other hand, the effects of viscous irrotational stress $\tau_{l}=2 \mu \nabla \otimes \nabla \phi$ within the solution domain is balanced internally, but in some boundaries such as bubble surface, it may be unbalanced and lead to power production [39]. The Joseph theory [25] suggest that in order to hold zero shear stress boundary conditions at the bubble boundary, pressure correction should be considered to compensate for non-zero irrotational shear stress. This parameter must be calculated such that the estimated power of the traction integral for VPF and VCVPF is the same at the bubble surface. For this purpose, the following statement is obtained from the mechanical energy equation for Navier-Stokes equations [27]:

$$
\int_{A} \mathbf{u . n}\left(-p_{v c}\right) d A=\int_{A} \mathbf{u . t} \tau_{l} d A .
$$

The values of $\tau_{l}$ and $p_{v c}$ at the collocation points are estimated on the bubble surface as follows:

$$
\begin{array}{r}
\sum_{j=1}^{M} \mathbf{u}_{j} \cdot \mathbf{n}_{j}\left(-p_{v c, j}\right) 2 \pi l_{j} R_{j} \sin \theta_{j} \\
=\sum_{j=1}^{M} \mathbf{u}_{j} \cdot \mathbf{t}_{j} \tau_{l, j} 2 \pi l_{j} R_{j} \sin \theta_{j}
\end{array}
$$

where $M$ is the number of elements on the bubble surface and $l_{j}$ is the length of the $j$ th element. In the above equation, $R_{j}$ and $\theta_{j}$ indicate radius and angle relative to $z$-coordinate in element $j$, respectively.

In this study, it is assumed that the power of traction integral in VPF method for each bubble level element is equal to the power of traction integral in VCVPF method for the same element. In other words, the viscosity difference due to uncompensated irrotational shear stress in each element is equal to the viscosity loss in that element. Therefore, simplifying Eq. (13) yields the following expression:

$$
p_{v c, j}=-\frac{\mathbf{u}_{j} \cdot \mathbf{t}_{j} \tau_{l, j}}{\mathbf{u}_{j} \cdot \mathbf{n}_{j}} .
$$

\subsection{Nondimensionalization}

Maximum bubble radius $R_{m}$, pressure difference driving the bubble collapse $\Delta p=p_{\infty}-p_{c}$, and fluid density are selected as the main nondimensionalizing parameters for the governing equations and numerical results. 


$$
\begin{aligned}
& {\left[\begin{array}{c}
\bar{R} \\
\bar{Z} \\
\bar{l}
\end{array}\right]=\frac{\left[\begin{array}{c}
R \\
Z \\
l
\end{array}\right]}{R_{m}}, \quad\left[\begin{array}{c}
\bar{\tau} \\
\bar{p}_{v c}
\end{array}\right]=\frac{\left[\begin{array}{c}
\tau \\
p_{v c}
\end{array}\right]}{\Delta p},} \\
& {\left[\begin{array}{c}
\bar{\psi} \\
\bar{\eta}
\end{array}\right]=\left[\begin{array}{c}
\psi \\
\eta
\end{array}\right]\left(\frac{\rho}{\Delta p}\right)^{0.5},} \\
& \lambda=\frac{R_{c y l}}{R_{m}}, \quad \bar{t}=\frac{t}{R_{m}}\left(\frac{\Delta p}{\rho}\right)^{0.5}, \\
& \bar{\mu}=\mu \frac{1}{\rho^{0.5} \Delta p^{0.5} R_{M}}, \quad \bar{\phi}=\frac{\phi}{R_{m}}\left(\frac{\rho}{\Delta p}\right)^{0.5}
\end{aligned}
$$

where $\lambda$ is the dimensionless channel radius, $\psi$ is the radial velocity, and $\eta$ is the tangential velocity. The nondimensional dynamic condition on the bubble surface is described as follows:

$$
\frac{D \bar{\phi}}{D \bar{t}}=1-\varepsilon\left(\frac{R_{i}}{R}\right)^{3 \gamma}+\frac{1}{2} \rho|\bar{\nabla} \bar{\phi}|^{2}+! \frac{2 \bar{k}}{W e}-\frac{2}{R e} \frac{\partial \bar{\psi}}{\partial \bar{n}}-\bar{p}_{v c}
$$

where $\varepsilon=p_{g 0} / \Delta p$ is the strength parameter. In Eq. (17), Weber number and Reynolds number are defined as $\mathrm{We}=\Delta p R_{m} / \sigma$ and $\operatorname{Re}=R_{m} \sqrt{\Delta p \rho} / \mu$, respectively. The nondimensional form of Eq. (13) can be formulated as:

$$
\begin{aligned}
& \sum_{j=1}^{m}\left(\bar{\psi}_{j} \bar{p}_{v_{j}}\right) 2 \pi \bar{l}_{j} \bar{R} \sin \theta_{j}= \\
& \quad \sum_{j=1}^{m}\left(\bar{\psi}_{j} \bar{\tau}_{s_{j}}+\bar{\eta}_{j} \bar{\tau}_{s_{j}}\right) 2 \pi \bar{l}_{j} \bar{R} \sin \theta_{j} .
\end{aligned}
$$

\subsection{Numerical implementation}

In this study, in order to implement BEM with threedimensional axisymmetric geometry, cylindrical coordinate system $(r, z)$ is used.

\subsubsection{Discretization}

A second-order function is used to express the vertical velocity in each node $(J)$ of the bubble surface based on two adjacent nodes $(j-1$ and $j+1)$.

$$
\phi_{j}=a S^{2}+b S+c
$$

where $a, b$, and $c$ are constants of the second-order equation. $S$ is the distance of the desired node $(j-1$ or $j+1$ ) from the node $j$. Now, by applying Eq. (19) to node $j+1$ or $j-1$, the magnitude of $S$ will be equal to the magnitude of $l_{j+1}$ or $l_{j-1}$, respectively.

The potential change to the bubble surface profile $(S)$ is obtained by [40]:

$$
\left(\frac{\partial \phi}{\partial S}\right)_{j}=\frac{\phi_{j+1} l_{j}^{2}-\phi_{j-1} l_{j+1}^{2}+\phi_{j}\left(l_{j+1}^{2}-l_{j}^{2}\right)}{l_{j} l_{j+1}\left(l_{j}+l_{j+1}\right)} .
$$

Solving the linear equation set in Eq. (1), the vertical velocity at collocation points can be calculated. The final position of elements after a small time scale, $\Delta t$, is obtained from the following equations:

$$
\begin{aligned}
& r_{j}^{t+\Delta t}=r_{j}^{t}+u_{j}^{t} \Delta t+O(\Delta t)^{2}, \\
& z_{j}^{t+\Delta t}=z_{j}^{t}+v_{j}^{t} \Delta t+O(\Delta t)^{2} .
\end{aligned}
$$

In order to calculate the velocity potential on the new time scale, the discretized form of the modified Bernoulli's equation is used:

$$
\begin{aligned}
\phi_{j}^{t+\Delta t}= & \phi_{j}^{t}+\frac{\Delta t}{\rho}\left\{\frac{1}{2} \rho|\nabla \phi|^{2}+\rho g z+\sigma k+p_{\infty}\right. \\
& \left.-p_{c}-p_{i g}\left(\frac{R_{i}}{R}\right)^{3 \gamma}-2 \mu \frac{\partial^{2} \phi}{\partial n^{2}}-p_{v c}\right\}^{t} .
\end{aligned}
$$

For more details about numerical implementations based on BEM, please refer to [40].

Nondimensional UISS and VPC on the bubble surface based on vertical and tangential velocities are calculated by:

$$
\begin{aligned}
& \bar{\tau}_{s_{j}}=2 \bar{\mu}\left[\frac{1}{2} \frac{\partial \bar{\eta}_{j}}{\partial n_{j}}-\frac{\bar{\eta}_{j}}{2 \bar{r}_{j}}+\frac{1}{2 \bar{r}_{j}} \frac{\partial \bar{\psi}_{j}}{\partial S_{j}}\right], \\
& \bar{p}_{v c, j}=-\frac{\bar{\psi}_{j} \bar{\tau}_{s_{j}}+\bar{\eta}_{j} \bar{\tau}_{s_{j}}}{\bar{\psi}_{j}} .
\end{aligned}
$$

Variation of tangential velocity in the vertical direction over the bubble surface can be estimated by the following equation:

$$
\frac{\partial \bar{\eta}_{j}}{\partial n_{j}}=\frac{\bar{\eta}_{j}^{i+1}-\bar{\eta}_{j}^{i}}{\Delta \bar{L}}
$$

where $\bar{\eta}_{j}^{i+1}$ shows the nondimensional tangential velocity considered at distance $\Delta \bar{L}$ from collocation point $j$ over the radial direction in fluid flow. For calculating the vertical velocity distribution over the bubble surface, cubic spline functions are employed. Therefore, changes in vertical velocity relative to the vector tangent to the bubble surface can be estimated as follows [40]:

$$
\frac{\partial \bar{\psi}_{j}}{\partial \bar{S}_{j}}=\frac{\bar{\psi}_{j+1} \bar{l}_{j}^{2}-\bar{\psi}_{j-1} \bar{l}_{j+1}^{2}+\bar{\psi}_{j}\left(\bar{l}_{j+1}^{2}-\bar{l}_{j}^{2}\right)}{\bar{l}_{j} \bar{l}_{j+1}\left(\bar{l}_{j}+\bar{l}_{j+1}\right)}
$$

\section{Numerical model verification}

\subsection{Independence of results from the number of nodes}

In order to investigate the independence of numerical results from the number of nodes on the bubble surface, variation of $V / V_{\max }$ against the dimensionless time at $\lambda=1.15$ was observed. By referring to Figure 2, it is evident that for a number of nodes greater than 18 , 


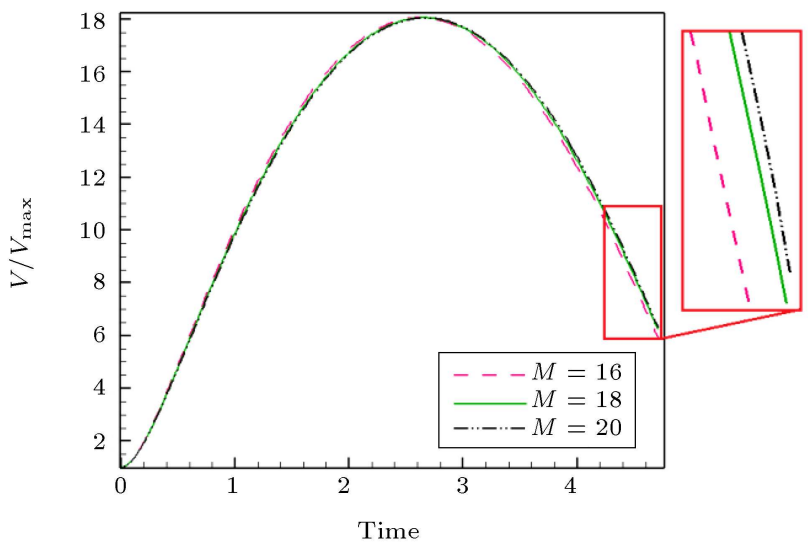

Figure 2. Independence of the results from the number of nodes.

the variation of $V / V_{\max }$ against the dimensionless time the is very small (eventually about $2 \%$ ). Therefore, the optimum number of nodes on the bubble surface, which results in the independence of the numerical results from the number of nodes, is assumed to be $M=18$.

\subsection{Comparison with the empirical results}

$\mathrm{Ni}$ et al. [14] conducted a series of experiments on bubble production by electric spark inside a narrow tube in water. Growth and collapse of the bubble were recorded by a high-velocity camera with the frame rate of 20000 frames/sec. The input parameters used in validation are presented in Table 1.

In Figure 3, the empirical results are compared with our numerical results. The figure shows that a good consistency exists between the empirical and numerical results. Figure 3(a) shows the spark bubble formation moment. In the empirical picture, at this stage, the bubble can be observed as a luminous domain. Figure $3(\mathrm{~b})$ shows the moment in which the bubble is at its maximum volume. The bubble cross-section at the expansion stage becomes elliptical since the cylindrical walls function as a barrier against bubble growth. At the end of expansion stage, the bubble velocity at the two ends of bubble along the channel axis is almost zero.

The high static pressure in the fluid around the

Table 1. Input parameters used in validation [14].

\begin{tabular}{lcc}
\hline Quantity [unit] & Symbol & Value \\
\hline Tube inner diameter & $D$ & 46 \\
Dimensionless channel radius [-] & $\lambda$ & 1.7 \\
Fluid surface tension $\left[\mathrm{kg} / \mathrm{s}^{2}\right]$ & $\sigma$ & $5.4 \times 10^{-5}$ \\
Fluid density [kg/ ${ }^{3}$ ] & $\rho$ & 998 \\
Environment pressure [kPa] & $p_{0}$ & 101.3 \\
Bubble maximum radius [mm] & $R_{m}$ & 0.001 \\
Fluid viscosity [kg/m.s] & $\mu$ & 100 \\
Strength parameter [-] & $\varepsilon$ & 18 \\
Number of collocation point-bubbles [-] & $M$ & 80 \\
Number of collocation point-tubes [-] & $N$ & \\
\hline
\end{tabular}

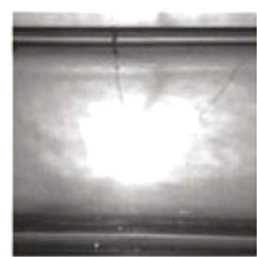

$t \approx 0 \mathrm{~ms}$

(a)

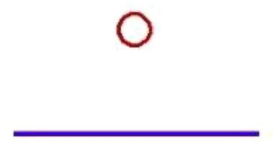

$t=0 \mathrm{~ms}$

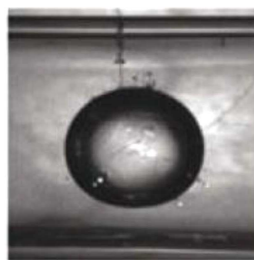

$t \approx 3.15 \mathrm{~ms}$

(b)

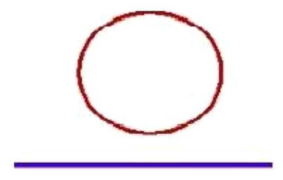

$t=3.15 \mathrm{~ms}$

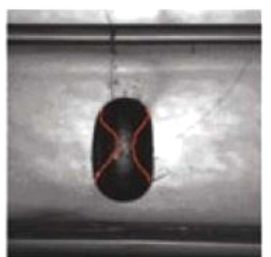

$t \approx 4.95 \mathrm{~ms}$

(c)

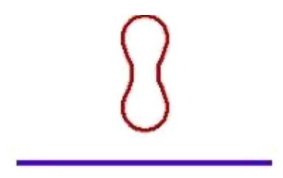

$t=4.96 \mathrm{~ms}$

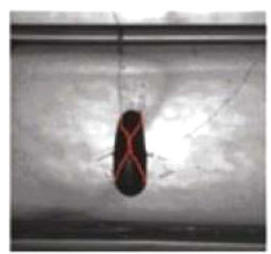

$t \approx 5.40 \mathrm{~ms}$

(d)

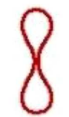

$t=5.42 \mathrm{~ms}$

Figure 3. Comparison between the present numerical results and experimental results reported by Ni et al. [14] at $\lambda=1.7$ and $\operatorname{Re}=134865$. 
two ends of bubble located along the channel axis directs the bubble towards the contraction stage and then, counter-jet emerges. According to Figure 3(c) and $(d)$, with penetration of the counter jet at the end of contraction stage, the bubble takes the shape of sand hour glass.

The oscillation period in the numerical results is slightly larger than that in the experiment results. Also, a small deviation in time scale between the empirical photos and the numerical model is observed. Error in measuring time and maximum bubble radius in experiments can be one of the most important reasons for the small difference between experimental and numerical results. In these experiments, considering the frame rate, the time between two recorded images is $0.05 \mathrm{~ms}$. In other words, the measurement accuracy of time scale in empirical experiments is $0.05 \mathrm{~ms}$. On the other hand, spatial resolution limitation of the camera causes errors in maximum bubble diameter estimation. The limitation in the number of pixels used in the photo structure makes image resolution dependent on the size of the pixels. Therefore, measuring the maximum diameter of the bubble is affected by the relative resolution of the image, which can be affected by an error.

\subsection{Comparison with the numerical results}

In order to validate the VCVPF method proposed in this study, the numerical results published by Minsier et al. [31] are used. They applied the volume of fluid method to bubble growth and collapse near the rigid wall inside the viscous fluid. In their numerical model, the dimensionless channel radius $\lambda=0.6$, oil viscosity $\mu_{\text {oil }}=0.05 \mathrm{~kg} /(\mathrm{m} . \mathrm{s})$, and the maximum bubble radius $R_{m}=1 \mathrm{~mm}$ were assumed. Figure 4 shows the comparison of numerical results obtained by the VCVPF method and volume of fluid model [31] for bubble dynamics in oil. Figure 4(a) reveals a slight difference between bubbles in the two models at the maximum volume. Also, according to Figure 4(b) to (d), the occurrence of phenomena such as bubble flattening near the wall, the formation of counter-jet at the same time, rapid jet penetration into the bubble, and almost similar time periods confirms the validity of our numerical results during the contraction stage.

\section{Results and discussion}

Table 2 summarizes the main input parameters used in the numerical model.

\subsection{Effect of viscosity and Reynolds number on bubble dynamics}

In this section, the effect of fluid viscosity on bubble dynamics inside a narrow channel is investigated by using the proposed numerical model. Bubble shapes for two viscosity values of $\mu_{\text {oil }}=0.05 \mathrm{~kg} /(\mathrm{m} . \mathrm{s})$ and $\mu_{\text {water }}=0.001 \mathrm{~kg} /(\mathrm{m} . \mathrm{s})$ at $\lambda=1.15$ are compared.

Table 2. Input parameters used in the numerical model.

\begin{tabular}{lcc}
\hline Quantity [unit] & Symbol & Value \\
\hline Bubble & & \\
Specific heat ratio [-] & $\gamma$ & 1.4 \\
Reference temperature [K] & $T_{\infty}$ & 293.15 \\
Reference pressure [kPa] & $p_{\infty}$ & 101.3 \\
Strength parameter [-] & $\varepsilon$ & 100 \\
Number of collocation points [-] & $M$ & 18 \\
Fluid & & \\
Density [kg/m ${ }^{3}$ ] & $\rho$ & 998 \\
Interface surface tension [kg/s $\left.{ }^{2}\right]$ & $\sigma$ & 0.0725 \\
Channel & & \\
Number of collocation points [-] & $N$ & 80 \\
\hline
\end{tabular}

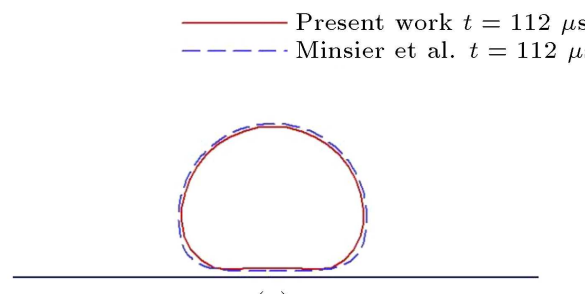

(a)

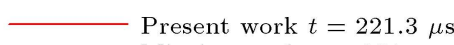

- - - - Minsier et al. $t=221 \mu \mathrm{s}$

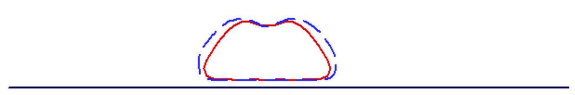

(c)

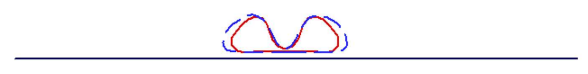

(d)

Figure 4. Comparison of the bubble shapes in oil with $\mu_{\text {oil }}=0.05 \mathrm{~kg} /(\mathrm{m} . \mathrm{s})$ at $\lambda=0.6$ between the present work and numerical results published by Minsier et al. [31]. 


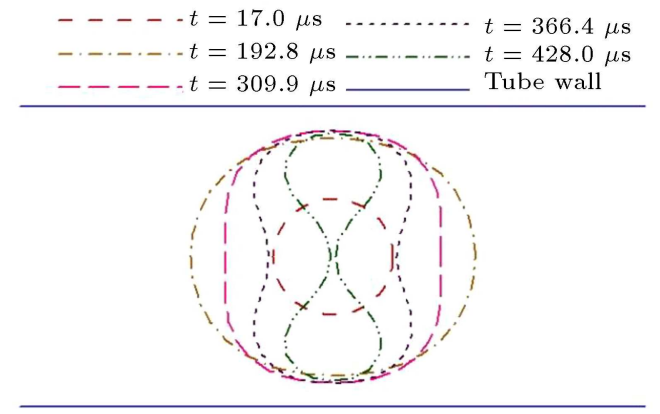

(a)

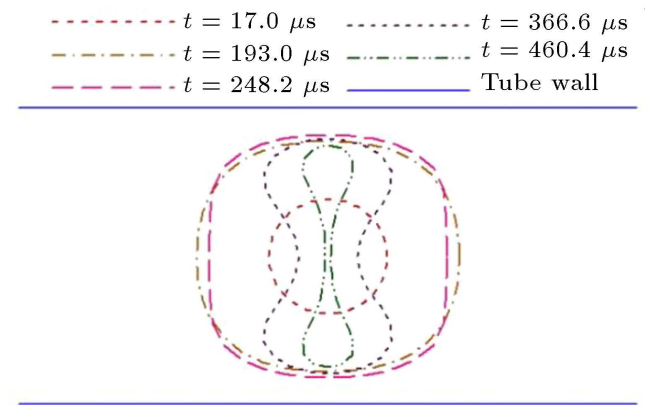

(b)

Figure 5. Evolution of bubble shape in various times in a narrow channel with $\lambda=1.15$ and $R_{m}=1 \mathrm{~mm}$ : (a) $\mu_{\text {water }}=0.001 \mathrm{~kg} /(\mathrm{m} . \mathrm{s}), \mathrm{Re}=10000$ and (b) $\mu_{\text {oil }}=0.05 \mathrm{~kg} /(\mathrm{m} . \mathrm{s}), \mathrm{Re}=200$.

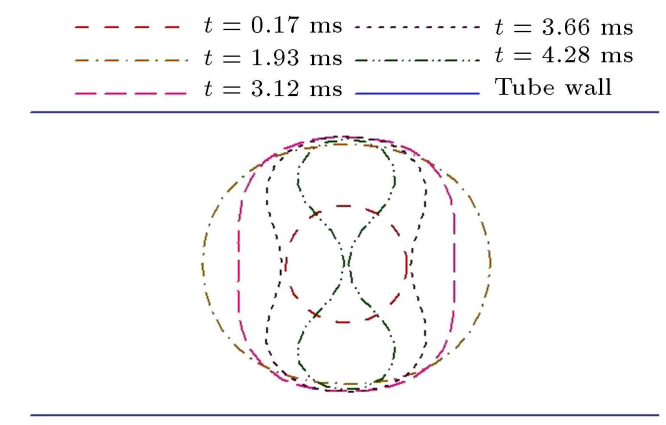

(a)

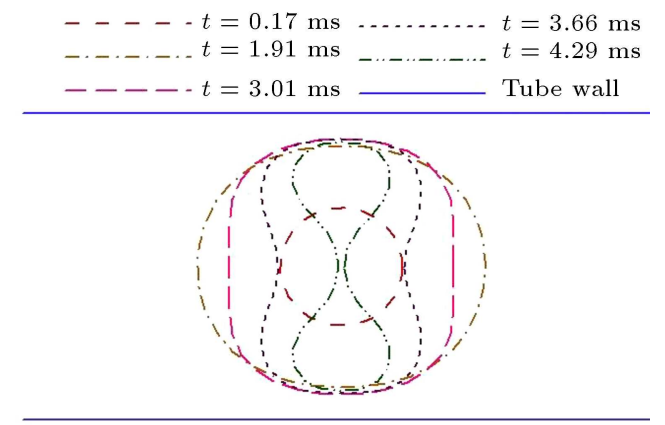

(b)

Figure 6. Evolution of bubble shape in various times in a narrow channel with $\lambda=1.15$ and $R_{m}=10 \mathrm{~mm}$ : (a) $\mu_{\text {water }}=0.001 \mathrm{~kg} /(\mathrm{m} . \mathrm{s}), \mathrm{R} e=100000$ and (b) $\mu_{\text {oil }}=0.05 \mathrm{~kg} /(\mathrm{m} . \mathrm{s}), \mathrm{Re}=2000$.

The results for two maximum bubble radii of $R_{m}=$ $1 \mathrm{~mm}$ and $R_{m}=10 \mathrm{~mm}$ are studied.

In Figure 5, the dynamic behavior of bubble for $R_{m}=1 \mathrm{~mm}$ at several dimensionless times is indicated. The required time for bubble growth up to the maximum radius in oil $(t=193.0 \mu \mathrm{s})$ is slightly larger than that in water $(t=192.8 \mu \mathrm{s})$. Therefore, it can be concluded that with an increase in viscosity, bubble growth becomes slower. The bubble volume for oil at the end of the growth stage is slightly lower than that for water. Thus, the bubble volume ratio, which is the ratio of bubble volume to its maximum volume, for oil and water is 83.1 and 83.7, respectively. Comparison of bubble shapes at the end of contraction stage indicates that the bubble volume further decreases with increase in viscosity. The bubble volume ratio at the end of the contraction stage for oil and water is 12.5 and 31.3 , respectively. This is justified because in both growth and contraction stages, with an increase in viscosity, most of the driving force is consumed to overcome viscous forces. For this reason, bubble growth is lower at the end of the growth stage. Also, at the end of the contraction stage, the bubble is flattened like a sandhour glass and its final volume becomes less.

In order to investigate the effect of Reynolds number, the results for $R_{m}=10 \mathrm{~mm}$ are presented in
Figure 6. As the figure indicates, at the growth stage, alike the contraction stage, no significant difference occurs in the volume and shape of the bubble between oil and water. Therefore, the ratio of bubble volume to its maximum volume at the end of the growth stage for oil and water is 83.65 and 83.70 , respectively. Also, the value of this parameter at the end of the contraction stage is 30.5 and 31.5 , respectively. This result can be explained as follows: an increase in maximum bubble radius results in a decrease in dimensionless viscosity Eq. (15) and consequently, according to Eq. (23), UISS is decreased. On the other hand, it can be seen that Reynolds number increases as the maximum bubble radius increases. According to Eq. (15), normal stress has a reverse relationship with Reynolds number. Therefore, with an increase in the Reynolds number and maximum bubble radius, the effect of viscosity on bubble life and shape decreases. The comparison of Figures 5 and 6 shows that when the Reynolds number is in the order of $O\left(10^{3}\right)$, the viscosity effects are greatly reduced and can be ignored.

\subsection{Effect of $\lambda$ on bubble dynamics}

The time history curves for bubble volume ratio to its maximum volume $\left(V / V_{\max }\right)$ at $\lambda=0.95, \lambda=1.15$, and $\lambda=1.35$ are drawn in Figure 7 . Initial time 


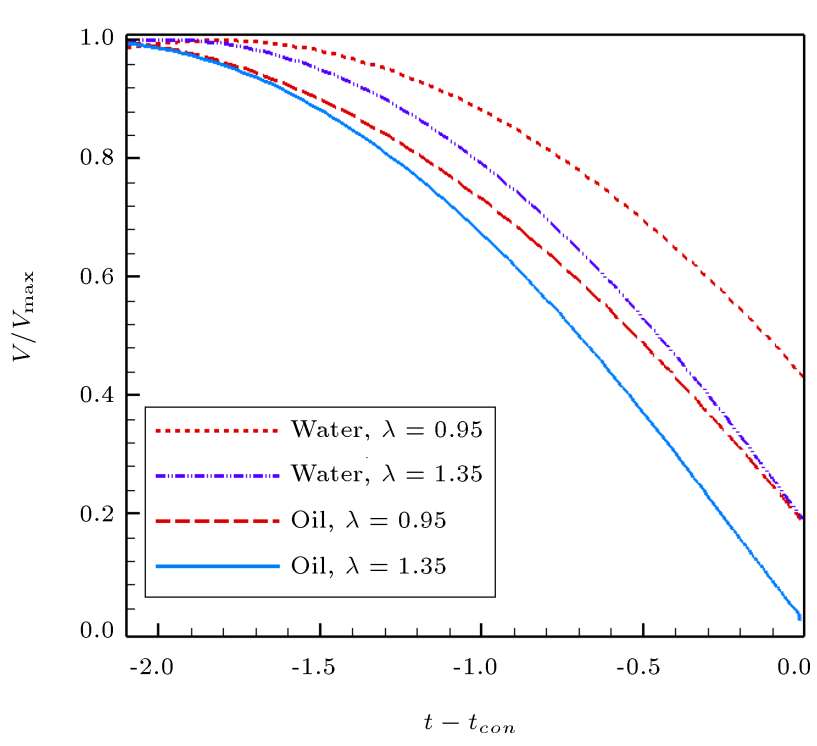

Figure 7. Variation of $V / V_{\max }$ as a function of dimensionless time.

and final time are related to the beginning and end of contraction stage. At all dimensionless channel radii, the value of $V / V_{\max }$ for the bubble in oil is less than that in water. At the end of the contraction stage, assuming $\lambda=0.95$, the magnitude of $V / V_{\max }$ for oil is approximately $53 \%$ less than that for water, while by assuming $\lambda=1.15$, it is $47 \%$. It means that during the contraction stage, the rate of bubble volume decrease for oil is always less than that for water at all $\lambda$ values. It is observed that higher viscosity brings about severe decrease in $V / V_{\max }$ at the minimum dimensionless radius parameter $(\lambda=0.95)$. This trend is justified noting that the decrease in $\lambda$ causes an increase in bubble shape deviation from the initial spherical shape. Therefore, the bubble is elongated in the direction of channel axis. This increases UISS over the bubble surface, further decreasing the bubble volume at the contraction stage.

Figure 8 depicts the maximum $z$-coordinate absolute value versus non-dimensional time. Given the

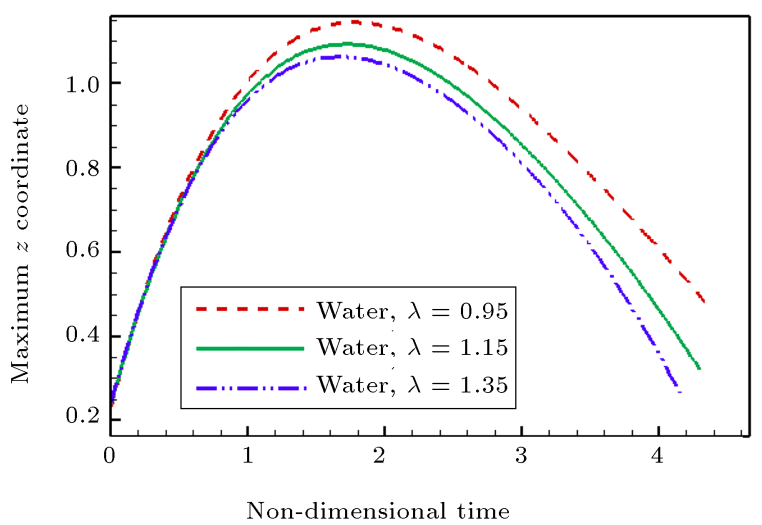

symmetry, it is obvious that $\mathrm{r}$-coordinate values for these points are almost zero. Also, Figure 8 reveals that with decrease in $\lambda$, the bubble is elongated and larger values of maximum $z$-coordinate occur at the end of the growth stage. Also, at similar $\lambda$, with an increased viscosity, maximum $z$-coordinate values decrease. In other words, viscosity flattens more bubbles, specifically the smaller ones. As an example, the maximum $z$-coordinate of the bubble boundary for oil is approximately $13 \%$ less than that for water. Undoubtedly, with the increase in the amount of $\lambda$, the difference will be reduced.

\subsection{The pressure field}

In order to fully understand the effect of viscosity on hydrodynamics of the bubble, path lines and pressure contours at different times are indicated in Figure 9 for two viscosities. As can be seen, the bubble behavior in both viscosities is almost the same. At first, the bubble rapidly grows due to the high pressure inside it and subsequently, with increase in its volume, the pressure decreases. Therefore, while near the end of the growth stage, the pressure inside bubble will be almost equal to the surrounding fluid pressure, it still grows through motion inertia. The direction of path lines in growth stage - Figure 9(a1) and (a2) and Figure 9(b1) and (b2) - confirms that the bubble behaves as a source actuating the fluid. The path lines in Figures 9 (a3) and (b3) indicate the fluid tendency towards creating two counter jets inside the bubble. The increase in pressure around the bubble near the symmetry axis leads to the rapid penetration of jets into the bubble. In the contraction stage, the direction of path lines is towards inside the bubble, which confirms the fact that bubble behaves as sink actuator of fluid - Figure 9(a4) and (b4). Also, it can be observed that with respect to the bubble lifetime, with increase in viscosity, the bubble reacts slowly. The bubble lifetime in the oil is $\bar{t}=4.570$, while the corresponding value in water is $\bar{t}=4.213$. According to Figure 9(b3), at the contraction stage in oil, the high-pressure zone surrounds most of the

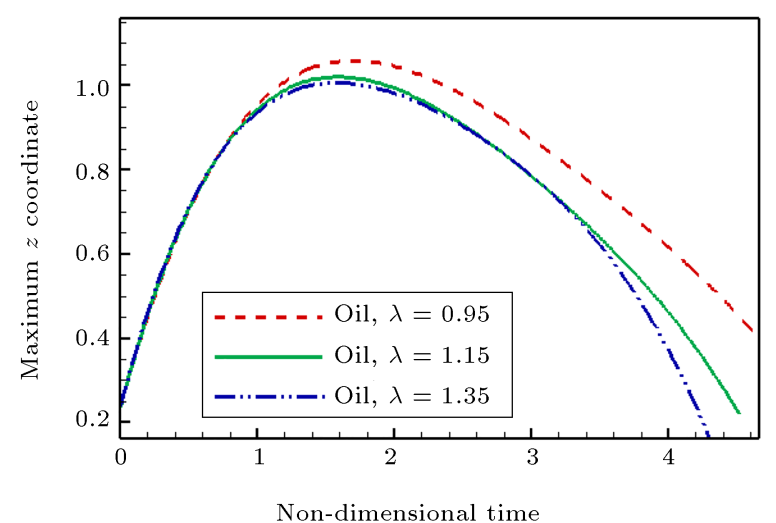

Figure 8. Maximum z-coordinate of bubble boundary versus the non-dimensional time with $\lambda=1.15$ and $\operatorname{Re}=200$. 

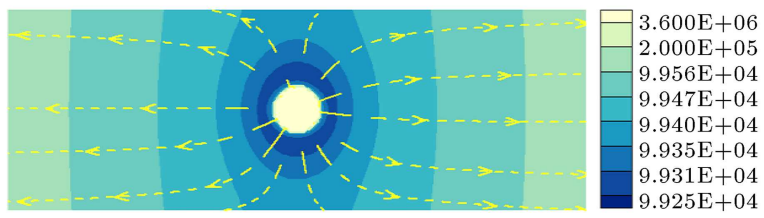

(a1) $\bar{t}=0.001$

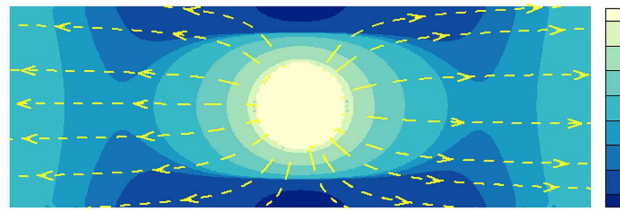

(a2) $\bar{t}=0.169$

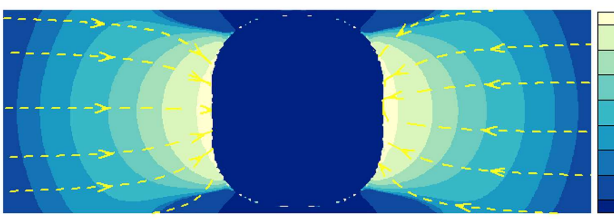

(a3) $\bar{t}=3.008$

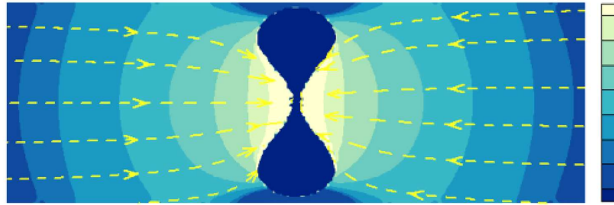

(a4) $\bar{t}=4.213$

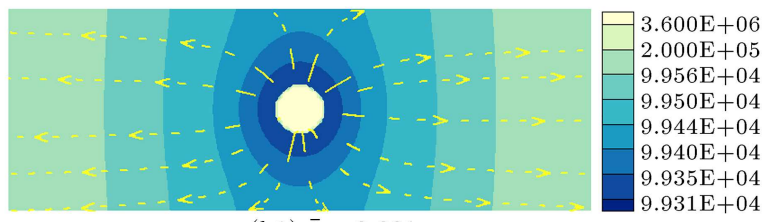

(b1) $\bar{t}=0.001$

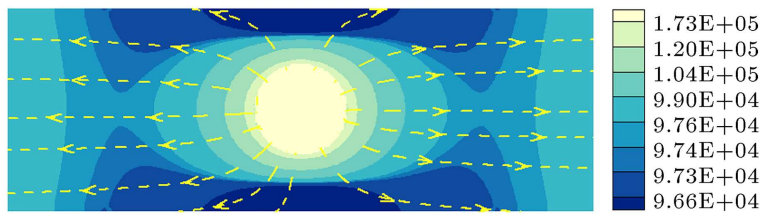

(b2) $\bar{t}=0.170$

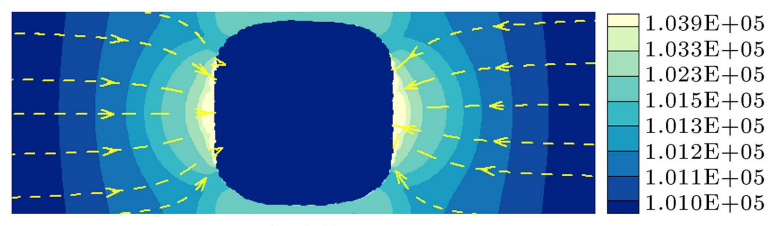

(b3) $\bar{t}=2.812$

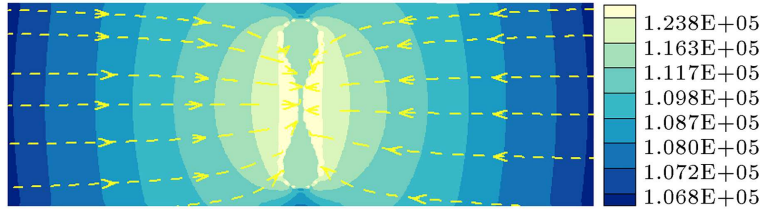

(b4) $\bar{t}=4.570$

Figure 9. Nondimensional pressure field and streamlines in the flow induced by the bubble evolution for $\lambda=1.15$ : (a) $\mu_{\text {water }}=0.001 \mathrm{~kg} /(\mathrm{m} . \mathrm{s}), \operatorname{Re}=10000$ and (b) $\mu_{\text {Oil }}=0.05 \mathrm{~kg} /(\mathrm{m} . \mathrm{s}), \operatorname{Re}=200$.

bubble surface while in water, the high-pressure zone occurs around the channel axis. Therefore, at the end of contraction stage, in the oil fluid, those parts of the bubble surface that are closer to the channel wall take a shrinking mode towards the bubble due to the pressure distribution and UISS effects. Referring to Figure 9(a4) and (b4), in the oil fluid, a further decrease in the bubble volume causes the pressure inside to be higher than that in water. The pressure inside the bubble at the end of contraction stage in oil is $115 \mathrm{kPa}$ and in water is $33 \mathrm{kPa}$.

\subsection{Analyzing viscosity component}

In Figure 10, the behavior of UISS, VPC, and viscous normal stress versus the dimensionless time are indicated in the third collocation point on the bubble surface. At the beginning of the growth stage, viscous normal stress experiences severe changes. After a rapid increase, it continues descending until the end of the growth stage so that at maximum bubble value, it is almost zero. With the formation of the counter-jet and its penetration into the bubble, viscous normal stress slightly increases. On the other hand, at the beginning of the bubble life, due to the completely spherical shape, UISS does not exist at the bubble surface and the zero value of this parameter at the zero moment confirms this reality. The value of this parameter increases at the beginning of the bubble

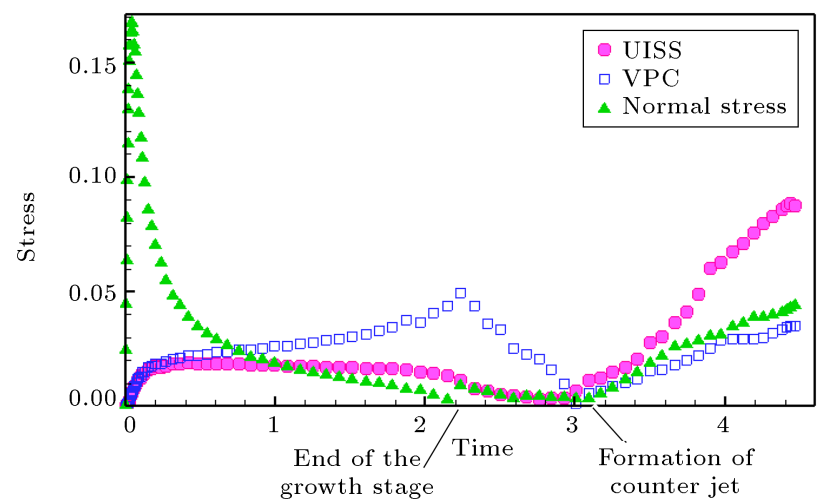

Figure 10. Evolution of Unbalanced Irrotational Shear Stress (UISS), Viscous Pressure Correction (VPC), and viscous normal stress as a function of the non-dimensional time in the third collocation point on the bubble surface with $\lambda=1.35$ and $\operatorname{Re}=200$.

lifetime and then, decreases until the formation of the counter-jet $\left(t_{j e t}\right)$. After $t_{j e t}$, with a change in bubble shape and severe deviation from the initial spherical shape, UISS value significantly increases. VPC value at the beginning of the growth stage is almost zero as well. During the growth stage, bubble shape deviation from spherical form gradually increases, which leads to an increasing VPC. After the formation of the counterjet, VPC increases slightly like UISS.

The velocity of the axis center point on the 


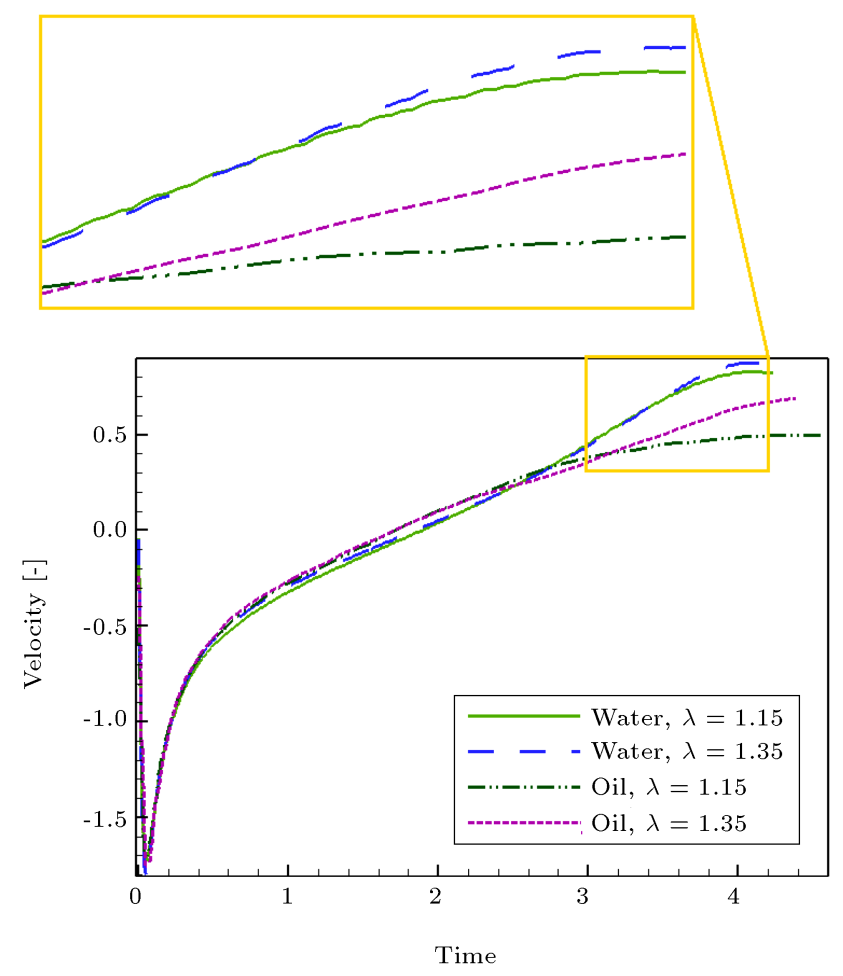

Figure 11. Time history of the non-dimensional velocity of axis center point on the bubble surface with $\lambda=1.35$ and $\operatorname{Re}=200$.

bubble surface is plotted versus the non-dimensional time in Figure 11. In this figure, the negative and positive values refer to the growth and contraction stages, respectively. It is noteworthy that at the growth stage, increasing viscosity does not exert a significant change in velocity. However, at the contraction stage, for a specific value of $\lambda$, velocity increases over time. At the expansion stage, the magnitudes of viscosity parameters are not significant. Therefore, by increasing viscosity, no significant difference occurs between the velocities of the interaction point of the axis of symmetry with the bubble surface. However, in bubble contraction, especially at the latest stages of bubble contraction that the viscosity terms significantly increase, a portion of the bubble driving force is used to overcome the viscosity forces. This causes a significant reduction in the velocity of the interaction point of the axis of symmetry with the bubble surface. Under the conditions of $\operatorname{Re}=200$ and $\lambda=1.15$, ratio of the rate of velocity reduction on the interaction point of the axis of symmetry with the bubble surface in oil to that in water is approximately $37 \%$. This reduction is equal to $22 \%$ in the case of $\lambda=1.35$.

Changes in the absolute value of viscous normal stress on the bubble surface with respect to time are shown in Figure 12. With symmetry, changes in half of the collocation points were investigated. As can be seen in this figure, at the growth stage $(\bar{t}<2.4)$, viscous

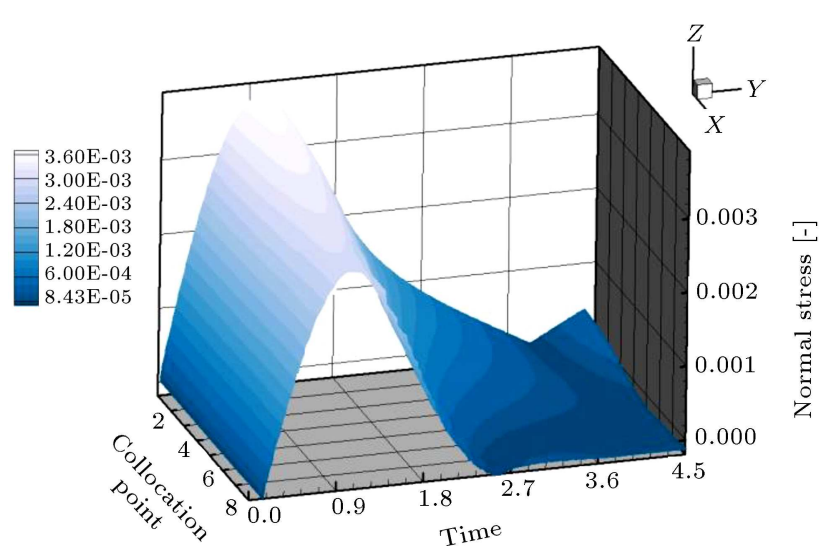

Figure 12. Viscous normal stress in collocation points on the bubble surface versus nondimensional time with $\lambda=1.15$ and $\operatorname{Re}=200$.

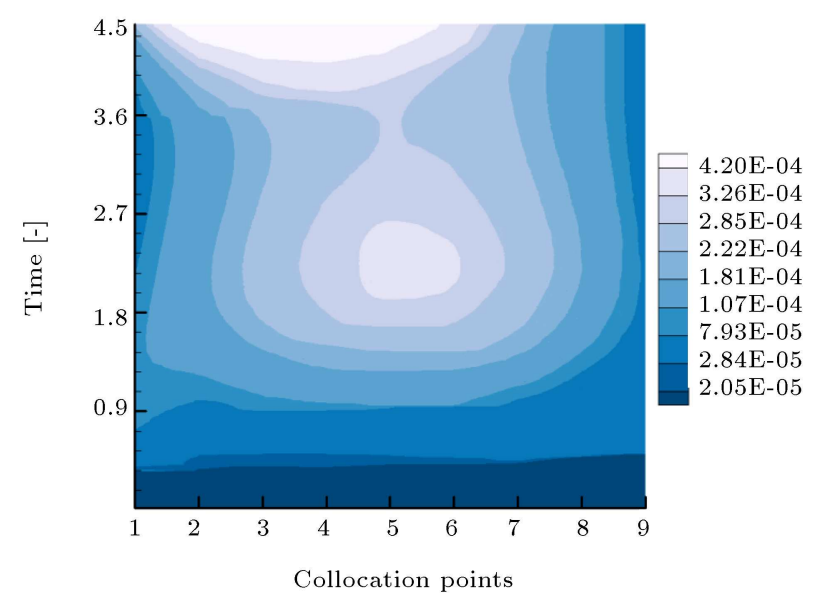

Figure 13. Contours of Unbalanced Irrotational Shear Stress (UISS) in collocation points on the bubble surface versus nondimensional time with $\lambda=1.15$ and $\operatorname{Re}=200$.

normal stress has maximum value in all collocation points. Extreme changes in the velocity of the early bubble growth stage result in a rapid increase in viscous normal stress. Then, with decrease in velocity variation, viscous normal stress shows descending behavior. As a result, in the contraction stage, even at near-wall points where changes in velocity are small, its value approaches zero. At a specific time, the normal stress value near the axis of symmetry is greater than that near the wall.

The variation of UISS in collocation points on the bubble surface as a function of time is illustrated in Figure 13. With the passage of time and near the end of the growth stage, the value of UISS increases in all collocation points. At this time, the maximum value of UISS occurs at intermediate collocation points. It is worth to note that at the end of the contraction stage, a significant increase in the UISS value is observed in most collocation points. This can be justified by the severe changes in tangential and normal velocities near the bubble surface. The maximum UISS value at the 
contraction stage is $6.70 \mathrm{E}-04$, which occurs at the end of bubble lifetime on node 3 .

By investigating Figures 12 and 13, the following results can be obtained.

The viscous normal stress reaches a maximum value in the growth stage only when the UISS experiences the maximum value at the end of the contraction stage as well as the end of growth stage. Also, the maximum value of viscous normal stress occurs in the nearest collocation point to the symmetry axis. However, the UISS at both stages is maximized at the intermediate collocation points. The shape of the bubble in both the expansion and contraction stages is influenced by the presence of the cylindrical wall and by the pressure at the far field. It is observed that the shape of intermediate nodes on the bubble surface is further changed over the bubble lifetime. This is due to the effects of far-field flow (near the cylindrical symmetry axis) and tube wall. In other words, extreme changes in the shape and physical parameters such as the pressure and velocity fields on the middle nodes of the bubble surface are the reason for higher values of UISS than those on the other nodes. It is observed that the viscous normal stress in all of the collocation points, after experiencing the maximum amount at the early stage of growth, has either a decreasing trend or negligible values. However, UISS amount, except in the early life of the bubble, is particularly significant at the middle collocation points of bubble surface. Given that VPC is considered for non-zero irrotational shear stress compensation, a direct relationship can be imagined between the numerical value of VPC and UISS. It can be concluded that behaviors of the viscous normal stress and VPC on the bubble surface during its evolution are not similar. In studies that have been done so far, in order to apply viscosity effects to the bubble dynamics, VPC is considered as a linear function of normal stress. However, the results of the present research given in Figures 12 and 13 show that the overall behaviors of these two parameters during evolution of the vapor bubble are not the same. Therefore, it should be noted that considering a relation between these two parameters over the bubble lifetime can increase the numerical error of the calculations. Eventually, considering consistency between VPC and normal stress [25-27] in certain cases is not necessarily appropriate.

\section{Conclusion}

A numerical model based on Boundary Element Method (BEM) was used to simulate the dynamics of a single bubble in a rigid narrow channel filled with a viscous liquid. The effects of viscosity were evaluated by applying the parameters of Unbalanced Irrational Shear Stress (UISS) and Viscous Pressure
Correction (VPC) through the Viscous Correction of Viscous Potential Flow (VCVPF) method.

The numerical results of the present study were verified by comparing them with the reported experimental results for the bubble profile and its lifetime when the bubble was inside a narrow channel filled with a viscous liquid [14] as well as when the bubble was in the vicinity of a flat rigid boundary [31]. The comparisons showed good agreement between the numerical results of this study and the reported experimental and numerical data.

It was found that by increasing the viscosity of liquid around the vapor bubble:

(a) The lifetime of bubble increased. As an example, under the conditions of $\operatorname{Re}=200$ and $\lambda=1.15$; the bubble lifetime for oil is $t=460.4 \mu$ s while for water is $t=428.0 \mu \mathrm{s}$;

(b) At the end of the contraction stage, the velocity of the bubble boundary decreased. As an example, under the conditions of $\operatorname{Re}=200$ and $\lambda=1.35$, the ratio of the rate of decrease in velocity at the interaction point of the axis of symmetry with the bubble surface in oil to the rate of decrease in velocity at the corresponding point in water was approximately $37 \%$;

(c) The bubble contracted slowly. For example, under the conditions of $\operatorname{Re}=200$ and $\lambda=1.35$, the bubble lifetime in oil was $7.8 \%$ higher than that in water;

(d) The final bubble volume decreased. As an example, at the end of the contraction stage, assuming $\lambda=0.95$, the magnitude of $V / V_{\max }$ for oil was approximately $53 \%$ less than that for water;

(e) The sand hour glass profile of the bubble at the end of its contraction stage was flattened.

In addition, it was concluded that for Reynolds numbers at the order of $O\left(10^{3}\right)$, the viscosity effects were extremely reduced and could be neglected. Moreover, the results indicated that viscous normal stress was maximized at all points of the bubble surface at the growth stage. However, the magnitude of UISS, except in the beginning of the bubble lifetime, was significant at the points of the bubble surface that were relatively near the internal wall of the narrow channel. Finally, it was found that the variations of viscous normal stress and VPC with respect to time on the surface of the bubble were not similar.

\section{References}

1. Guo, X., Du, Z., Li, G., et al. "High frequency vibration recovery enhancement technology in the heavy oil fields of China", SPE International Thermal 
Operations and Heavy Oil Symposium, Bakersfield, California, 16-18 March, SPE-86956, pp. 1-18 (2004).

2. Xu, H. and $\mathrm{Pu}, \mathrm{C}$. "Removal of near-wellbore formation damage by ultrasonic stimulation", Pet. Sci. Technol., 31, pp. 563-571 (2013).

3. Jin, Y., Zheng, X., Chu, X., et al. "Oil recovery from oil sludge through combined ultrasound and thermochemical cleaning treatment", Ind. Eng. Chem. Res., 51, pp. 9213-9217 (2012).

4. Massoud, E.Z., Xiao, Q., El-Gamal, H.A., et al. "Numerical study of an individual Taylor bubble rising through stagnant liquids under laminar flow regime", Ocean Eng., 162, pp. 117-137 (2018).

5. Amani, E., Ahmadpour, A., and Tohidi, M. "A numerical study of the rise of a Taylor bubble through a sudden/gradual expansion in Newtonian and shearthinning liquids", Scientia Iranica, 152, pp. 236-246 (2019).

6. Zhang, S., Wang, S.P., Zhang, A.M., et al. "Numerical study on motion of the air-gun bubble based on boundary integral method", Ocean Eng., 154, pp. 7080 (2018).

7. Ziolkowski, A. "Measurement of air-gun bubble oscillations", Geophys., 63, pp. 2009-2024 (1998).

8. Chahine, G.L., Hsiao, Ch.T., Choi, J.K., et al. "Bubble augmented water jet propulsion: two-phase model development and experimental validation", 27th Symposium on Naval Hydrodynamics, Seoul, Korea, 5-10 October, pp. 1-17 (2008).

9. Wu, X., Choi, J.K., Singh, S., et al. "Experimental and numerical investigation of bubble augmented water jet propulsion", J. Hydraulics, 24, pp. 635-647 (2012).

10. Hayati, A.N., Hashemi, S.M., and Shams, M. "Design and analysis of bubble-injected water ramjets with discrete injection configurations by computational fluid dynamics method", Proc. IMechE Part C: J. Mechanical Engineering Science, 227, pp. 1945-1955 (2015).

11. Makaloski, V., Rohlffs, F., Konstantinos, S., et al. "Bubble counter for measurement of air bubbles during thoracic stent-graft deployment in a flow model", $J$. Surg. Res., 232, pp. 121-127 (2018).

12. Sánchez, R.M., Rivero, F., Bastante, T., et al. "Intracoronary bubbles: Iatrogenic air embolism assessed with optical coherence tomography", JACC Cardiovascular Interventions, 10, pp. 153-154 (2017).

13. Farhangmehr, V., Hajizadeh, A., Shervani-Tabar, M.T., et al. "Numerical investigation on the pulsating bubble dynamics in a narrow cylinder with a compliant coating", Fluid Dyn. Res., 46, 015513, pp. 1-27 (2014).

14. Ni, B.Y., Zhang, A.M., Wang, Q.X., et al. "Experimental and numerical study on the growth and collapse of a bubble in a narrow tube", Acta Mech. Sin., 28, pp. 1248-1260 (2012).

15. Baradaran-Fard, M. and Nikseresht, A.H. "Numerical simulation of unsteady $3 \mathrm{D}$ cavitating flows over axisymmetric cavitators", Scientia Iranica, 19, pp. 12651278 (2012).
16. Hanafizadeh, P., Saidi, M.H., Nouri Gheimasi, M.H., and Ghanbarzadeh, S. "Experimental investigation of air-water, two-phase flow regimes in vertical mini pipe", Scientia Iranica, 18, pp. 923-929 (2011).

17. Shervani-Tabar, M.T. and Rouhollahi, R. "Numerical study on the effect of the concave rigid boundaries on the cavitation intensity", Scientia Iranica, 24, pp. 1958-1965 (2017).

18. Fayzi, P., Bastani, D., Lotfi, M., and Chamangiz Khararoodi, M. "The effects of bubble detachment shape on rising bubble hydrodynamics", Scientia Iranica, 26(3), pp. 1546-1554 (2018). DOI: 10.24200/sci.2018.51823.2383.

19. Ory, E., Yuan, H., Prosperetti, A., et al. "Growth and collapse of a vapor bubble in a narrow tube", Phys. Fluids, 12, pp. 1268-1277 (2000).

20. Shervani-Tabar, M.T. and Eslamian, A. "Dynamics of a vapour bubble inside a vertical rigid cylinder", 16th Australasian Fluid Mechanics Conference Crown Plaza, Gold Coast, Australia, 2-7 December, pp. 14201426 (2007).

21. Miao, H., Gracewski, S.M., and Dalecki, D. "Ultrasonic excitation of a bubble inside a deformable tube: Implications for ultrasonically induced hemorrhage", J. Acoust Soc. Am., 124, pp. 2374-2384 (2008).

22. Clanet, Ch., Heraud, P., and Starby G. "On the motion of bubbles in vertical tubes of arbitrary crosssections: some complements to the Dumitrescu-Taylor problem", J. Fluid Mech., 519, pp. 359-376 (2004).

23. Moore, D.W. "The boundary layer on a spherical gas bubble", J. Fluid Mech., 16, pp. 161-176 (1963).

24. Kang, I.S. and Leal, L.G. "The drag coefficient for a spherical bubble in a uniform streaming flow", Phys. Fluids, 31, pp. 233-237 (1988).

25. Joseph, D.D. "Potential flow of viscous fluids: historical notes", Int. J. Multiphase Flow, 32, pp. 285-310 (2006).

26. Klaseboer, E., Manica, R., Chan, D.Y.C., et al. "BEM simulations of potential flow with viscous effects as applied", Eng. Anal. Boundary Elem., 35, pp. 489-494 (2011).

27. Joseph, D.D. and Wang, H. "Dissipation approximation and viscous potential flow", J. Fluid Mech., 505, pp. $365-377$ (2004).

28. Manmi, K. and Wang, Q. "Acoustic microbubble dynamics with viscous effects", Ultrason. Sonochem., 36, pp. 427-436 (2016).

29. Zhang, A.M. and Ni, B.Y. "Three-dimensional boundary integral simulations of motion and deformation of bubbles with viscous effects", Comput. Fluids, 92, pp. 22-33 (2014).

30. Wang, S.P., Wang, Q.X., Leppinen, D.M., et al. "Acoustic bubble dynamics in a microvessel surrounded by elastic material", Phys. Fluids, 30, 012104, pp. 1-10 (2018). 
31. Minsier, V., Wilde, J.D., and Proost, J. "Simulation of the effect of viscosity on jet penetration into a single cavitating bubble", J. Appl. Phys., 106, 084906, pp. $1-10$ (2009).

32. Taib, B.B. "Boundary integral method applied to cavitation bubble dynamics", PhD Thesis, University of Wollongong, Australia (1985).

33. Mehravarana, M. and Kazemzadeh, S. "Simulation of buoyant bubble motion in viscous flows employing lattice Boltzmann and level set methods", Scientia Iranica, 18, pp. 231-240 (2011).

34. Lind, S.J. and Philips, T.N. "The effect of viscoelasticity on the dynamics of gas bubbles near free surfaces", Phys. Fluids, 25, 022104, pp. 1-32 (2013).

35. Katsikadelis, J.T. "Preliminary mathematical concept", In Boundary Elements: Theory and Applications, 1st Ed., UK, Elsevier Science, p. 16 (2002).

36. Zhou, J., Hu, J., and Yuan, Sh. "Modeling bubble evolution in air-oil mixture with a simplified method", Proc. IMechE Part C: J. Mechanical Engineering Science, 230, pp. 2865-2871 (2016).

37. White, F.M. "Fundamental equations of compressible viscous flow", In Viscous Fluid Flow, 2nd Ed., New York, McGraw-Hill, pp. 61-69 (1991).

38. Batchelor, G.K. "Flow at large Reynolds number: effects of viscosity", In An Introduction to Fluid Dynamics, 1st Ed., UK, Cambridge University Press, pp. 264-377 (2000).

39. Joseph, D.D., Funada, T., and Wang, J. "Helmholtz decomposition coupling rotational to irrotational flow", In Potential Flows of Viscous and Viscoelastic Fluids, 1st Ed., UK, Cambridge University Press, pp. 15-18 (2007).

40. Shervani-Tabar, M.T. "Computer study of a cavity bubble near a rigid boundary, a free surface and a compliant wall", $\mathrm{PhD}$ Thesis, University of Wollongong, Australia (1995).

\section{Biographies}

Amir Soroureddin is a $\mathrm{PhD}$ candidate in the Mechanical Engineering Department of Tabriz University, Iran. He received his BSc and MSc degrees in Mechanical Engineering from Tabriz University in 2008 and 2011, respectively. He is currently a lecturer at Azarbaijan Shahid Madani University in Tabriz, Iran. The main focus of his thesis is investigation of bubble dynamics in porous medium with viscous fluid based on viscous correction of viscous potential flow theory. His fields of interests are cavitation, bubble dynamics, and designing and exergoeconomic analyses of energy converting systems.

Mohammad-Taghi Shervani-Tabar received the $\mathrm{PhD}$ degree in Mechanical Engineering from University of Wollongong in 1995. He is currently a professor at University of Tabriz. His main interests are in the areas of cavitation and bubble dynamics, boundary element method, and computational fluid dynamics. His interest extends to the field of droplet management, fluid flow in porous media (oil recovery enhancement), and impinging jets. Professor Shervani-Tabar has published more than 45 publications.

Habib Aminfar was born in 1948 in Iran. After received his BSc in Mechanical Engineering in 1970 from Sharif University of Technology. He pursued his studies in the same field at Shiraz University for the MSc degree and University of Columbia Mo. (Jointly with MIT) for the $\mathrm{PhD}$ degree and received the degrees in 1973 and 1980, respectively. During his studies, he offered several courses such as two-phase flow and convective heat transfer. Currently, he works as a full-time faculty member at University of Tabriz. His research interests are two-phase flow, convection, conduction, and radiative heat transfer in nano-fluids. 